\title{
Tympanostomy Tube Complication in Otitis Media with Effusion \\ ${ }^{1}$ Ghassan Hassan Rahim, ${ }^{2}$ Mohammed Rahim Ali
}

\section{ABSTRACT}

Background: Otitis media with effusion is characterized by accumulation of fluid in the middle ear in absence of acute inflammation and it is the most common cause of acquired hearing loss in children, and may negatively affect language development failure of medical treatment of middle ear effusion frequently require myringotomy and tympanostomy tube insertion.

Objectives: To determine tympanostomy tube complications of tube in children with chronic otitis media with effusion who were treated with Shah Grommet tube insertion.

Methods: The Medical records of 162 ears of 87 children (52 male and 35 female) were reviewed respectively, the patients ages were between 3 to 16 years old (mean age $=8.11$ years), patient were followed for 6-66 months (mean 23.3) after tympanostomy tube insertion. Tube extrusion time was also reviewed in all patients, and the indication for surgery was chronic middle ear effusion.
Results: Otorrhea accured in nine ears $(5.6 \%)$, granulation tissue was seen in 2 ears $(1.2 \%)$, myringosclerosis in $(34.6 \%)$ persistent perforation $(5.6 \%)$, atrophy $(23.5 \%)$ retraction $(16.7 \%)$ and medial displacement $1.2 \%$ the average extrusion time was 8.5 month $( \pm 4.6)$.

Conclusions: complications of tympanostomy tube insertion are common and the most common are otorrhea myringosclerosis, atrophy but they are generally insignificant consequently in majority of these complications there is no need for management.

Keywords: Otitis media with effusion ,tymanostomy tube shah type.

\section{${ }^{1}$ Corresponding author :}

Ghassan H Rahim F.I.C.Ms D.L.O Al-Kindy Teaching Hospital, Surgery Department

Received at 10/6/2018

Accepted at 3/2/2019

\section{INTRODUCTION}

Otitis media with effusion (OME is characterized by accumulation of fluid in the middle ear, in the absence of acute inflammation. The condition is the most common cause of acquired hearing loss in children and may negatively affect language development. Failure of medical treatment of middle ear effusion frequently requires myringotomy and tympanostomy tube insertion (1). This operation is one of the most common surgical procedures and usually requires general anesthesia in children although otolaryngologists consider conservative treatment as first line of management of otitis media with effusion ${ }^{(2)}$.

Many types of tympanostomy tubes are available commercially. But, complication rates of various tubes have been reported ${ }^{(3-7)}$. The most common complications of tympanostomy tube insertion were otorrhea, atrophy and myringosclerosis ${ }^{(2-8)}$. In this study, we tried to determine the specific complications of Shah Grommet tympanostomy tube insertion.

\section{METHODS}

In this study, patients with chronic OME who underwent tympanostomy tube insertion in the Department of Otorhinolaryngology of AL-Kindy Teaching Hospital between July 2008 and November 2013 were reviewed retrospectively. In all cases the indication for operation was chronic OME. The diagnoses were done by otoscopic examination, tympanometric and puretone audiometric measurements before operation. During otoscopy any retraction, increased vascularization, tympanic membrane dullness or loss of opalescence, fluid levels, or air bubbles were recorded. Patients with otoscopic signs of OME, type B tympanogram and any conductive hearing loss were considered positive for OME, Operation was performed on patients whose OME, lasted more than 3 months, all cases were examined postoperatively at 1 week and 1 month.

Further control examinations have been made in our clinic once in every 3 months until extrusion of tubes, After extrusion of tubes, patient with perforation and retraction were continued to be followed up for 3 month periods.

In this study, the operated cases were called for control between January 2014and May 2014 in order to determine 
presence or absence of complications after tympanostomy tube extrusion, final control otomicroscopic examinations, tympanometric and audiometric evaluations were performed on all patients. Two cases with craniofacial anomalies Down's syndrome and cleft palate, ten cases that underwent only myringotomy instead of tympanostomy tube insertion and seven cases that could not be called for control were excluded from the study, Also 11 patients whose tympanostomy tube was still in place were excluded from the study because we can't determine complication occurrence until extrusion grommets. Patient's age, sex, surgical procedure performed, duration of tympanostomy tube in place and complications were recorded. Follow up period which was defined as the period

\section{DISCUSSION}

Tympanostomy tube insertion is a commonly performed surgical operation in children. various tympanostomy tubes have been used in the operation Short term tubes are Shepard, Armstrong, Shah, Sheey, Reuter Bobbin, Donaldson and Paparella type I, although long term tubes are Paparella type I, Butterfly, Per-Lee, andGoode T-tubes ${ }^{(3)}$.

Otorrhea is one of the most common complications after tympanostomy tube insertion, The incidence varies from 0.8 to $8.3 \%(4,9)$ Early otorrhea occurs within 24 weeks postoperatively, and delayed otorhea seems more frequent and occurs after first month postoperatively ${ }^{(5)}$. AhTye et al. ${ }^{(9)}$ showed an incidence reaching $74.8 \%$ after 12 months and $83 \%$ after 18 months of the children who developed one or more episodes of otorrhea. Kay et al. ${ }^{(3)}$ found early otorrhea in $16 \%$ of patients and delayed otorrhea in $26 \%$ of patients in the meta-analysis. Long-term tubes are generally associated with a higher incidence of otorrhea. The incidence of otorrhea rates were: $32.5 \%$ for long-term tubes, and $14.8 \%$ for short-term tubes ${ }^{(3)}$. Several factors were blamed in the literature for the occurrence of otorrhea such as younger age, contamination from the external auditory canal during Surgery, upper respiratory tract infection or between the insertion time and final control time, was 6-66 months (median $23.3 \pm 14.9$ ).

\section{RESULT}

The study was completed with 162 ears of 87 children. They are 52 boys $(59.8 \%)$ and 35 girls (40.2\%) with ages ranging between 3 and 16 years (mean age $8.1 \pm$ 3.1 years). Unilateral tympanostomy tube insertion was performed in 12 patients $(13.8 \%)$. In 80 patients (92\%) adenoidectomy or adenotonsillectomy were performed with tympanostomy tube insertion. Nine patients (10.3\%) had multiple tympanostomy tubes placed while 78 patients $(89.7 \%)$ had first tubes placed. Seven patients had second tubes placed and two patients had third tubes placed. In

infected middle ear effusions (4,5,10) Otorrhea is not serious in most cases. The management of otorrhea usually include a topical preparation of otic drops containing an antibiotic steroid combination with or without oral antibiotics, after culture /sensitivity test of discharge a related meta- analysis found a $52 \%$ reduction of post-tympanostomy tube otorrhea when antibiotic prophylaxis was used $^{(11)}$. In this study, 5.6\% of ears had otorrhea. Otorrhea occurred early in most of the ears within the first 4 weeks.

All of them were treated with combination of systemic antibiotic and topical antibiotic-corticosteroid drugs. The mean incidence rate of granulation tissue occurring after tympanostomy tube insertion is less than 5\% (3). This complication rate was $0.3 \%$ for Shepard tubes, and $13 \%$ for T-tubes ${ }^{(4)}$. It is a predisposing factor for otorrhea ${ }^{(5)}$ Medical treatment includes the use of topical antibiotic steroid combination with or withoutoral antibiotics or topical chemical cauterization with silver nitrate solution ${ }^{(5)}$. In more serious cases the tube may need removal (1.8\%) ${ }^{(3)}$. In this study, granulation tissue was Seen in two ears $(1.2 \%)$, and treated with combination of systemic antibiotic and topical antibiotic corticosteroid drugs.

Perforation can be seen after surgical or spontaneous tube extrusion, and the rate of 
perforation in spontaneously extruded tubes (12) The majority of these perforations heal spontaneously. while persistent perforation was reported as a approximately $2.2 \%$ for short-term tubes and $16.6 \%$ for long-term tubes ${ }^{(3)}$. In other studies, the perforation rate was found in $16 \%$ for Paparella type $23 \%$ for Reuter bobbin, $45 \%$ for Sheehy, and 35\% for Ttube ${ }^{(12)}$. In the same study, the perforation rate was noted as $24 \%$ for children with a history of less than 3 sets of tubes, while it was $42 \%$ for children with a history of 3 or more sets of tubes ${ }^{(12)}$. They found that prolonged ventilation tube retention beyond 36 months resulted in an increased perforation rate after Surgical removal, In our study in only one ear of nine ears with persistent perforation was after second tympanostomy tube insertion was performed, while in the other eight ears with persistent performation first tympanostony tube insertion were performed. To treat persistent performation after tube removal different types of patching materials including paper, absorbable gelatin film, and fat plug were used.

Satio et al. (13) showed a decrease from 13.2 to $3.3 \%$ when using paper patching However, Nichols et al. ${ }^{(12)}$ and El-Bitar et al. (14) did not find any benefits from patching. In our study, perforation was found in $8 \%$ of ears, but, persistent perforation was in $5.6 \%$ of ears We did not perform patching to any of them, further surgery is planned on all of them.

Myingosclerosis signifies an increase in collagen fibrils in the lamina propria, with hyaline degeneration and calcification and is a frequent complication of tympanostomy tube insertion, Foreign body reaction, fibrous hyperplasia and inflammatory changes due to the tube, haemorrhage and the liberation of free haemoglobin between the layers of the tympanic membrane after myringotomy are blamed for the development of tympanosclerosis after insertion of a tympanostomy tube (15). In this study, myringosclerosis was the most common complication of tympanostomy tube insertion and the myringosclerosis incidence rates was $34.6 \%$ were similar to 32 and $40.4 \%$ reported by Kay et al. ${ }^{(3)}$ and
Johnston et al. ${ }^{(8)}$, respectively, It was usually located on the inferior quadrants of tympanic membrane, we did not detect any hearing loss due to myringosclerosis (by PTA \& Tympanometry).

A trophy may contribute to more serious complications, such as spontaneous perforations $(5,16)$. Minor atrophic scars and thickening of the pars tensa of tympanic membrane are more related to middle ear disorders, while Segmental atrophy seems to be directly related to the tube insertion ${ }^{(5,16)}$. In Johnston et al ${ }^{(8)}$ and Daly et al. ${ }^{(6)}$ studies the most common complication of tympanostomy tube insertion was atrophy of tympanic membranc, and was present in 74.7 and $67 \%$, respectively, Maw and Bawden ${ }^{(16)}$ found that $22 \%$ of tubed ears and $5 \%$ of nontubed ears had localized pars tensa atrophy at 10 years of follow-up .

In our study, atrophy rate was $23.5 \%$, and this rate was similar to $24.6 \%$ of the metaanalysis by Kay et al. ${ }^{(3)}$. We did not find any hearing loss.

Retraction rate was reported as $38 \%$ at 6 years of follow up (6). Diacova and McDonald ${ }^{(17)}$ found that $32 \%$ of tube ears and $28 \%$ of conservatively treated ears had complete retractions. According to our finding the incidence of retraction (16.7\%) was lower than these studies.

Cholesteatoma is extremely rare due to tympanostomy tube insertion ${ }^{(3,5)}$, but it is the most serious complication after tympanostomy tube insertion our cases had no cholesteatoma. Medial displacement of tympanostomy tubes are very rare ${ }^{(3},{ }^{18,19)}$. Meta-analysis indicated a $0.5 \%$ rate of medial displacement of tympanostony tubes ${ }^{(3)}$. Green et al. ${ }^{(19)}$ reported three cases of medial displacement of T-tubes. They did not remove the T-tubes in two cases because there was no effusion and conductive hearing loss in the follow-up examinations, In the other case removed the T-tube because of effusion and conductive hearing loss, we determined in two ears (1.2\%) of two patients with medial displacement of grommets, We did not remove the grommet in one of them because the patient was asymptomatic In the other one an effusion was observed and the grommet was removed. 


\section{CONCLUSION}

Complications of tympanostomy tube insertion are common Myringosclerosis, tympanic membrane atrophy and otorrhea are most frequently shown of these complications. But they are generally insignificant consequently, in the majority of these complications there is no need for the management.

\section{REFRENCES}

1.Bluestone CD Studies in otitis media: Children's Hospital of Pittsburgh University of Pittsburgh progress report 2004.Laryngoscope, (2004), 114:1-26

2. Talmon Y, Gadban H, Samet A, Gilbey P, Letichevsky V Medium-term middle ear ventilation with self-manufactured polyethylene $\mathrm{T}$-tubes for the treatment of children with middle ear effusion, JLarygol Otol, (2001), 115:699-703

3. Kay Dy, Nelson M, Rosenfeld RM Metaanalysis of tympanostomy tube sequelae. Otolaryngol Head Neck Surg, (2001) , 124:374-380

4. Kalcioglu MT,Cokkeser Y, Kizilay A, Ozturan O Follow up of 366 cas after tympanostomy tube insention: why is it draining? Otolaryngol Head Neck Surg, (2003) , 128:560-564

5. Vlastarakos PV, Nikolopoulos TP, Korres S, Tavoulari E,Tzagaroulakis A, Ferekidis E Grommets in otitis media with effusion: the most frequent operation in children But is it associated with significant complications? Eurn JPediatr, (2007) , 166385-391

6. Daly KA, Hunter LL, Lindgren BR, Margolis R, Gicebink GS Chronic otitis media with effusion sequelae in children treated with tubes, Arch Otolaryngol Head Neck Surg, (2003) , 129:517- 522

7. Lindstrom DR, Reuben B, Jacobson K, Flanary VA, Kerschner JE Long-term results of Armstrong beveled grommet tyImpanostony tubes in children Laryngoscope, (2004), 490 :114-194

8. Johnson LC, Feldman HM, Paradise JL, Bernard BS, Colbom DK Casselbrant MI Janosky JE Tympanic membrane abnomalities and heaning levels at the ages of 5 and 6 years in relation to pensistent otitis media and tympanostomy tube insertion in the finx 3 years of life: a prospective study incorporating arandomized clinical trial. Pediatrices , (2004), 114(1):e58-e67.

9. Ah- Tye C Paradise Colborn DK,Otorhea in young children after tympanostony-tube placement for persistent middle-ear effusion: prevalence incidence, and duration, Pediatrics, 2001,107:1251-1258

10.Scott BA Strunk CL : Jr Posttympanostomy otorrhea: the efficacy of canal presentation Laryngoscope, (1992), 102:1103-1107.

11. Hochman J, Blakley B, Abdoh A, Alcid H Post-tympan stomy tube otorhea a meta analysis. Otolaryngol Head Neck Surg, (2006) , 135:8-11.

12. Nichols PT, Ramadan HH, Wax MK, Santrock RD Relationship between tympanic membrane performations and retained ventilation tubes, Arch otolaryngol Head Neck Surg, (1998), 124:417- 419

13. Saito T, Iwaki B, Kohno Y, Ohtsubo T, Noda I, Mori s, Yanamoto T, shibamori Y, Saito H Prevention of per sistent ear drum perforation after long-term ventilation tube treatment for otitis media with effusion in children, Int Pediatr Otorhinolaryngol, (1996) , $38: 31-39$.

14. El-Bitar MA, Pena MT, Choi SS, Zalzal GH Retained ventilation tubes: should they be removed at 2 year? Arch Otorlaryngol Head Neck Surg, (2002), 128:1357-1360.

15. Koc A, Uneri C Sex distribution in children with tympanosclerosis after insertion of a tympanostomy tube, Eur Arc Otorhinolaryngol, (2001), 258:16-19.

16. Maw AR, Bawden R Tympanic membrane atrophy. scarring, atelectasis and attic retraction in persistent, untreated otitis media with effusion and following ventilation the insertion, Int $\mathbf{J}$ Pediatr Otorhinolaryngol, (1994), 30:189-204

17. Diacova S, Mc Donald TJA comparison of outcomes following tympanostomy tube placement or conservative measures for management of otitis media with effusion, Ear Nose Throat, (2007), J86:552-554

18. Kumar M, Khan AM, Davis s Medial displacement of grommets: an unwanted sequel of grommet insertion, J Laryngol Otol, (2000) , 114:448449.

19. Green KM, de Carpentier JP, Curley JW An unusual complication of Tubes, JLaryngol Otol, (1997), 111:282-283.

20.Rosenfeld RM, Culpepper L, Doyle KJ, et al: American Academy of pediatrics Subcommittee on otitis Media with Effusion ; American Academy of family physicians; American Academy of otolaryngology- head and Neck Surgery.clinical practice guideline : otitis media with effusion. otolaryngol head Neck Surg, 2004,130(5)(suppl): S95S118. 